\title{
University clinic and private practice treatment outcomes in Class I extraction and nonextraction patients: A comparative study with the American Board of Orthodontics Objective Grading System
}

\author{
Mislik, Barbara ; Konstantonis, Dimitrios ; Katsadouris, Alexios ; Eliades, Theodore
}

\begin{abstract}
INTRODUCTION The aim of this study was to compare treatment outcomes in university vs private practice settings with Class I patients using the American Board of Orthodontics Objective Grading System. METHODS A parent sample of 580 Class I patients treated with and without extractions of 4 first premolars was subjected to discriminant analysis to identify a borderline spectrum of 66 patients regarding the extraction modality. Of these patients, 34 were treated in private orthodontic practices, and 32 were treated in a university graduate orthodontic clinic. The treatment outcomes were evaluated using the 8 variables of the American Board of Orthodontics Objective Grading System. RESULTS The total scores ranged from 10 to 47 (mean, 25.44; SD, 9.8) for the university group and from 14 to 45 (mean, 25.94; SD, 7.7) for the private practice group. The university group achieved better scores for the variables of buccolingual inclination (mean difference, 2.28; 95\% confidence interval [CI], 0.59, 3.98; P = 0.01 ) and marginal ridges (mean difference, $1.32 ; 95 \% \mathrm{CI}, 0.28,2.36 ; \mathrm{P}=0.01$ ), and the private practice group achieved a better score for the variable of root angulation (mean difference, $-0.65 ; 95 \%$ CI, -1.26 , $-0.03 ; \mathrm{P}=0.04$ ). However, no statistically intergroup differences were found between the total American Board of Orthodontics Objective Grading System scores (mean difference, $-0.5 ; 95 \%$ CI, -3.82, 4.82; P = 0.82). CONCLUSIONS Patients can receive similar quality of orthodontic treatment in a private practice and a university clinic. The orthodontists in the private practices were more successful in angulating the roots properly, whereas the orthodontic residents accomplished better torque control of the posterior segments and better marginal ridges.
\end{abstract}

DOI: https://doi.org/10.1016/j.ajodo.2015.10.012

Posted at the Zurich Open Repository and Archive, University of Zurich

ZORA URL: https://doi.org/10.5167/uzh-133229

Journal Article

Accepted Version

Originally published at:

Mislik, Barbara; Konstantonis, Dimitrios; Katsadouris, Alexios; Eliades, Theodore (2016). University clinic and private practice treatment outcomes in Class I extraction and nonextraction patients: A comparative study with the American Board of Orthodontics Objective Grading System. American Journal of Orthodontics and Dentofacial Orthopedics, 149(2):253-258.

DOI: https://doi.org/10.1016/j.ajodo.2015.10.012 


\section{University clinic and private practice treatment outcomes in Class I extraction and non-extraction patients: A comparative study with the American Board of Orthodontics objective grading system.}

\section{$\underline{\text { Mislik } \mathrm{B}^{1}}, \underline{\text { Konstantonis } \mathrm{D}^{2}}$, Katsadouris $\mathrm{A}^{3}$, Eliades $\mathrm{T}^{4}$.}

- $\quad{ }^{1}$ Resident, Clinic of Orthodontics and Paediatric Dentistry, Center of Dental Medicine, Faculty of Medicine, University of Zurich, Zurich, Switzerland.

- ${ }^{2}$ Research associate, Department of Orthodontics, School of Dentistry, University of Athens, Athens, Greece.

- ${ }^{3}$ Postgraduate student, Department of Orthodontics, School of Dentistry, University of Athens, Athens, Greece.

- ${ }^{4}$ Professor and director, Clinic of Orthodontics and Paediatric Dentistry, Center of Dental Medicine, Faculty of Medicine, University of Zurich, Zurich, Switzerland. Electronic address: theodore.eliades@zzm.uzh.ch.

Am J Orthod Dentofacial Orthop. 2016 Feb;149(2):253-8. doi: 10.1016/j.ajodo.2015.10.012.

\section{ABSTRACT}

Aim: The aims of this study were to compare treatment outcomes in university vs private practice, Class I patients, by using the American Board of Orthodontics objective grading system (ABO-OGS).

Materials and Method: A parent sample of 580 Class I patients treated with or without extractions of four first premolars was subjected to discriminant analysis in order to identify a borderline spectrum of 66 patients regarding to the extraction modality. Of these patients, 34 were treated in private orthodontic practices and 32 in a university graduate orthodontic clinic. The treatment outcomes were evaluated using the 8 variables of the ABO-OGS.

Results: The total score ranged from 10 to 47 (mean, 25.44; SD 9.8) for the university group and from 14 to 45 (mean, 25.94; SD 7.7) for the private practice group. The university group achieved better scores in the variables of buccolingual inclination (mean difference 2.28; 95\% $\mathrm{Cl} 0.59,3.98 ; P=0.01$ ) and marginal ridges (mean difference 1.32; 95\% $\mathrm{Cl} 0.28,2.36$; $P=0.01)$ and the private practice group achieved better score in the variable of root 
angulation (mean difference $-0.65 ; 95 \% \mathrm{Cl}-1.26,-0.03 ; P=0.04$ ). However, no statistically intergroup differences were found between the total ABO-OGS scores (mean difference, $0.5 ; 95 \% \mathrm{Cl},-3.82,4.82 ; P=0.82)$.

Conclusions: Patients can receive similar quality of orthodontic treatment in a private practice or in a university clinic. Orthodontists in a private practice were more successful in angulating the roots properly whereas orthodontic residents accomplished better torque control of the posterior segments and better marginal ridges.

\section{Introduction}

The choice of treatment provider is an important concern when patients seek treatment. Fulfilled expectation regarding curative outcomes is one of the main determinants in a treatment's provider choice ${ }^{1}$. Other parameters like socioeconomic background, distance from the clinic and the level of clinical facilities play also an important role in a patient's decision process. A very limited number of research studies have assessed differences between orthodontic treatment outcomes derived in university clinic settings and in private practices. $^{2,3}$ Therefore, it remains still unclear whether the clinical skills of a private orthodontist can outweigh the detailed and supervised practice of a university graduate student.

The assessment of success of an orthodontic treatment involves ideally the evaluation of a patient's post treatment records. However, without a valid and reliable evaluation method it is rather difficult and often subjective to assess treatment outcomes. Since the 1970 s several indices that aim to assess objectively orthodontic treatment outcomes have been introduced. ${ }^{4-7}$ The peer assessment rating (PAR) index, introduced by Richmond in 1992 focused mainly on the degree of improvement a case exhibited. Specifically, it evaluated the malocclusion improvement between the initial and final situation but it did not measure tooth positions and occlusal results with precision. Subsequently, the 
index of complexity outcome and need (ICON), was introduced by Daniels and Richmond in $2000 .^{7}$ The ICON index evaluated the case complexity, the need of orthodontic treatment, the improvement of malocclusion as well as the treatment outcome. Among the main advantages of the ICON index were the objectivity of the treatment assessments; its simplicity and the lack of need for any special equipment. The main drawback of the aforementioned index was that esthetic considerations constituted the most important part of the evaluation.

The American Board of Orthodontics (ABO) recommended a more solid way of evaluating treatment outcomes ${ }^{8}$. The ABO-OGS uses a specific instrument in measuring dental casts while final panoramic radiographs are also evaluated by visual inspection (Fig.1). In detail, the ABO-OGS index rates the final occlusion with eight criteria, which contribute to ideal intercuspation and function. Best occlusion and alignment are present with a score of 0 points. For each parameter that deviates from ideal, 1 or 2 penalty points are added. Cases are classified as 'successful' or 'failed' according to their ABO-OGS score. A case with a total score of 20 points or less passes the ABO examination and one with more than 30 points fails. Cases scoring between 20-30 are subject to individual reassessment.

Consequently, high percentage of accordance can be achieved in both inter- and intra-examiner assessment. In recent orthodontic literature the ABO-OGS has been widely used in comparing treatment outcomes of mutually exclusive treatment approaches or techniques. ${ }^{9-12,13,14}$ In addition to being an objective clinical examination tool it has been also used to increase the reliability, validity and precision of the assessment of treatment progress and final outcome. ${ }^{7,15-16}$

The purpose of this study was to compare the results of orthodontic treatment in private practice with those provided by graduate residents in a university orthodontic clinic. Furthermore, we investigated whether the gender or age of the patient have an impact on the treatment quality.

\section{Materials and Methods}


An initial sample of 580 Class I patients was gathered from $X X X X X X X$ graduate orthodontic clinic and 5 private orthodontic practices in XXXXXXXX. Of these patients 329 were treated in the university clinic and 251 in the private practices whereas 427 patients received non-extraction treatment and 153 patients were treated with removal of 4 first premolars. The orthodontists who treated the cases in private practices had at least 15 years of clinical experience. The inclusion criteria for the sample were Caucasian male or female with a Class I skeletal and dental malocclusion, full complement of teeth excluding the third molars, no previous orthodontic treatment and no patients with dentofacial deformities or clefts. Furthermore, all patients were treated with preadjusted edgewise appliance in both arches without the use of temporary anchorage devices of any form. The records used in this study were plaster dental casts, panoramic and lateral cephalometric x-rays, whereas a complete set of diagnostic records was required for a case to be included in the study. All lateral cephalometric $x$-rays were taken in natural head position and were analyzed using the ViewBox (version 4.0.1.7; dHAL Software).

To obtain a subsample a discriminant analysis was utilized and a borderline sample that could have been treated either extraction or non-extraction was identified. By the use of the discriminant analysis it was ensured that all cases of the borderline sample exhibited the same degree of dental and skeletal discrepancy at the onset of treatment. In order to secure an accurate representation of all the dental, skeletal and soft-tissue traits that have an impact on the orthodontist's treatment decision the discriminant analysis encompassed a large set of data consisting of 26 cephalometric, 6 model and 2 demographic variables. Each patient received a discriminant score that ranged from -3.45 to 3.16 and the optimal cut off score according to which the cases are discriminated into the extraction or non-extraction group was determined at 0 . As patients' discriminants scores draw away from zero to the negative values, they are predicted as extraction and when they reach positive values they are predicted as non-extraction. With regards to the extraction modality, the borderline cases were selected around the cut off score. The fact that all these cases had similar discriminant 
scoring secured a matched sample. Statistical analysis in this study was carried out using SPSS software (version 19.0; IBM, Armonk, NY).

Because of the absence of any orthodontic anomaly requiring particular clinical skills to be addressed these patients sought treatment in one of the two orthodontic clinical settings (University clinic or private practice) with no anticipated particular preference to either of them. Furthermore, a considerable number of clinicians treated the cases, circa 20 residents in the university clinic and 12 different orthodontists in the private practices. Subsequently, the elimination of selection or proficiency bias was ensured.

A power test was calculated in order to assess the sample size required. The power test resulted in a sample size of 60 individuals needed to detect a clinically significant difference in the total score of 5 units with a common standard deviation of 7 units, assuming a two-sided type I error of $5 \%$ and an $80 \%$ power.

The final sample consisted of 66 Class I patients, which were considered borderline regarding the extraction modality (Table 1 ). Of these patients, 34 were treated in private orthodontic practices and 32 in the university clinic and $41(62.1 \%)$ were female and 25 (37.9\%) were male. Of the university clinic patients, 19 (59.4\%) were female and $13(40.6 \%)$ were male. Of the private practice patients 22 (64.7\%) were female and 12 (35.3\%) were male. The mean patient age was 14.88 (SD 7.36) and 17.06 (SD 7.87) for the university and the private practice group, respectively. Of the university patients, 10 were treated extraction whereas 22 received non-extraction treatment. Of the private practice patients, 20 were treated extraction and 14 received non-extraction treatment. The treatment outcomes were evaluated using the 8 variables of the ABO-OGS: alignment, marginal ridges, buccolingual inclination, overjet, occlusal contacts, occlusal relationships, interproximal contacts and root angulation. Initially, the principal investigator (BM) completed the necessary calibration process as instructed by the ABO. Then, the measurements were obtained using the special ABO gauge. 
To examine the intergroup differences between the scores of the eight ABO-OGS variables as well as between the total ABO-OGS scores for the university and the private practice group, descriptive and inferential statistics were performed and t-tests for independent samples were used (Table 2). The significance level was predetermined at $5 \%$ $(P \leq 0.05)$. Evaluation was performed for both random and systematic error of the method. Initially, Dahlberg's formula was used to assess intraexaminer repeatability according to the equation $D=\sqrt{\left(\sum d\right)^{2} / 2 \mathrm{~N}}$ where $\mathrm{D}$ is the difference between the pairs of replicate measurements. With the aid of a table of random numbers ten cases were randomly selected - 5 from each group- and re-evaluated 5 days later by the same examiner (BM). Values ranged from 0.224 to 0.922 thus revealing very good intra-examiner agreement, while the $d$ for the overall score was 1.830 .

Next, the intraclass correlation coefficient (ICC) was employed to evaluate interexaminer agreement. The ABO-OGS variables of 5 university and 5 private-practice cases were evaluated by an independent examiner, a member of the National Board of Orthodontics, and compared with the measurements of the main investigator. The ICC (2.2) of all measurements was 0.983 thus revealing outstanding agreement.

\section{Results}

The results of descriptive and inferential statistics calculated for the 8 ABO-OGS variables for the private practice and the university groups are shown in Table 2 . The total ABO-OGS scores ranged from 14 to 45 (mean, 25.94; SD, 7.69) for the private practice group and from 10 to 47 (mean, 25.44; SD, 9.81) for the university group. No statistically significant difference was found in the total ABO-OGS scores between patients treated in the university or in the private practice (mean difference, $-0.5 ; 95 \% \mathrm{Cl}-3.82,4.82 ; P=0.82$ ).

The progression of criteria for the university group from the highest mean score to the lowest mean score was alignment, occlusal contacts, buccolingual inclination, occlusal relationships, overjet, marginal ridges, root angulation, and interproximal contacts. A different sequence was found in the private practice group where the progression of criteria from the 
highest to the lowest mean score was buccolingual inclination, alignment, occlusal contacts, marginal ridges, overjet, occlusal relationships, root angulation, and interproximal contacts (Fig 2).

The university group achieved statistically significant better scores in the variables of buccolingual inclination (mean difference 2.28; $95 \% \mathrm{Cl} 0.59$, 3.98; $P=0.01$ ) and marginal ridges (mean difference 1.32; 95\% $\mathrm{Cl} 0.28,2.36 ; \mathrm{p}=0.01$ ). The private practice group achieved statistically significant better score in the variable of root angulation (mean difference $-0.65 ; 95 \% \mathrm{Cl}-1.26,-0.03 ; P=0.04)$. No statistically significant differences were found between the scores of the other 5 ABO-OGS variables. In both groups, interproximal contacts received the lowest scores: 0.16 for the university group and 0.15 for the private practice group (mean difference $-0.01 ; 95 \% \mathrm{Cl}-0.23,0.21 ; P=0.93$ ). Buccolingual inclination received the highest score in the private practice group (5.94, mean difference $2.28 ; 95 \% \mathrm{Cl}$ $0.59,3.98 ; P=0.01$ ) whereas alignment received the highest score in the university group (5.41, mean difference $-0.29 ; 95 \% \mathrm{Cl}-1.73,1.16 ; P=0.69)$.

\section{Discussion}

In a retrospective research survey comparing treatment outcomes of weakly matched cases may introduce susceptibility bias, since the differences detected at the outcome could possibly be attributed to pre-existing differences at the onset of treatment ${ }^{17,18}$ To address this issue, in the present study, the choice of discriminant analysis through which the borderline sample was obtained to ensure that all cases exhibited the same degree of dental and skeletal discrepancy at the onset of treatment. Furthermore, it was decided to include extraction as well as non-extraction borderline Class I cases since it has been previously reported that both treatment modalities can achieve similar level of treatment results. ${ }^{19}$ Additionally, from the patients' charts it was shown that no radically dissimilar treatment appliances and auxiliaries like mini implants, plates or extraoral devices common in addressing severe Class II or Class III anomalies were utilized. The large number of 
clinicians who treated the cases eliminated also the possibility of selection or proficiency bias.

No statistically significant differences in the total $A B O$ scores between patients treated in the university or in the private practice were revealed in this study. The total ABOOGS mean scores were 25.44 (SD 9.81) for the university group and 25.94 (SD 7.69) for the private practice group. These findings are in accordance with the results reported by Cook et al. but not with those reported by Yang-Powers et al. who found significant differences in favor of the private practice group. Cook et al. ${ }^{2}$ investigated 139 Class II Division 1 premolar extraction cases and reported no significant difference in the total score; 25.14 (SD, 11.87) in the university group and 25.97 (SD 9.70) in the private practice group. However, the variable of root angulation was omitted. Conversely, Yang-Powers at al. comparing 92 cases treated in a university educational program and 32 cases treated by private clinicians and previously presented to the $\mathrm{ABO}$ reported a significant difference between the total scores of the two groups; 45.54 (SD 18.33) and 33.88 (SD 9.69), $P<0.05$ respectively. $^{2}$

The results of this investigation indicate that the university group received significantly better scores for buccolingual inclination and marginal ridges. Interestingly, buccolingual inclination scored the highest in the private practice group. According to Yang-Powers at al. this variable, which assesses the torque of the posterior segments, seems to trouble orthodontists the most both in university and in private practice. The authors suggested that the lack of proper torque control is related to the difficulty in either recognizing or correcting the problem. Since in both dental arches, marginal ridges of adjacent posterior teeth should be at the same level the score difference between the 2 groups suggests that better bracket and band placement took place in the university clinic. However, Cook et al. reported better score for marginal ridges in the private practice group.

The private practice group showed significantly better root angulation than the university group. This finding is in accordance with Yang-Powers at al. that also reported this variable of achieving a low score of significant difference in both groups. In this research both groups achieved the lowest scores for interproximal contacts thus suggesting that closing 
spaces is a simple performance for orthodontists, an outcome also confirmed in other comparable studies $^{192,3,12}$. Different variables showing statistically significant difference between private practice and university groups have also been suggested: occlusal contacts, ${ }^{1-2}$ interproximal contacts, ${ }^{1}$ and overjet. $^{2}$

As shown in this research study orthodontic patients can choose to be treated in either a university clinic or a private practice having alike possibilities of achieving a favorable result. The orthodontic community comprising of private practitioners, residents and instructors can greatly benefit from an objective appraisal of the orthodontic treatment results. Additionally, through similar investigations clinicians can consider and improve the orthodontic tooth positioning goals that seem to concern them the most.

\section{Conclusions}

The results of this investigation suggest that orthodontic patients have the same probability of receiving an acceptable treatment result in a private practice as well as in a university clinic. However, while the total ABO-OGS score between the 2 groups did not show any statistically significant difference, the university group achieved better scores in the variables of buccolingual inclination and marginal ridges whereas the private practice group proved to be more efficient in angulating the roots properly. Still, neither the treatment provider nor the age or gender of the patients was a significant predictor of treatment quality.

\section{Highlights}

-Treatment results were compared between private practices and a university clinic.

-The American Board of Orthodontics Objective Grading System was used to assess outcomes.

-The probability of acceptable treatment was the same and unrelated to setting.

-The university group had better scores for buccolingual inclination and marginal ridges.

-The private practice group was more efficient in angulating the roots properly. 


\section{References}

1. Victoor A, Delnoij DM, Friele RD, Rademakers JJ. Determinants of patient choice of healthcare providers: a scoping review. BMC Health Serv Res 2012;12:272.

2. Cook DR, Harris EF, Vaden JL. Comparison of university and private-practice orthodontic treatment outcomes with the American Board of Orthodontics objective grading system. Am J Orthod Dentofacial Orthop 2005;127:707-712.

3. Yang-Powers LC, Sadowsky C, Rosenstein S, BeGole EA. Treatment outcome in a graduate orthodontic clinic using the American Board of Orthodontics grading system. Am J Orthod Dentofacial Orthop 2002;122:451-455.

4. Summers CJ. The occlusal index: a system for identifying and scoring occlusal disorders. Am J Orthod 1971;59:552-567.

5. Andrews LF. The six keys to normal occlusion. Am J Orthod 1972;62:296-309.

6. Pickering EA, Vig P. The occlusal index used to assess orthodontic treatment. $\mathrm{Br} \mathrm{J}$ Orthod 1975;2:47-51.

7. Daniels C, Richmond S. The development of the index of complexity, outcome and need (ICON). J Orthod 2000;27:149-162.

8. Casko JS, Vaden JL, Kokich VG, Damone J, James RD, Cangialosi TJ et al. Objective grading system for dental casts and panoramic radiographs. American Board of Orthodontics. Am J Orthod Dentofacial Orthop 1998;114:589-599.

9. Chaison ET, Liu X, Tuncay OC. The quality of treatment in the adult orthodontic patient as judged by orthodontists and measured by the Objective Grading System. Am J Orthod Dentofacial Orthop 2011;139:S69-75.

10. Hsieh TJ, Pinskaya Y, Roberts WE. Assessment of orthodontic treatment outcomes: early treatment versus late treatment. Angle Orthod 2005;75:162-170.

11. Kuncio D, Maganzini A, Shelton C, Freeman K. Invisalign and traditional orthodontic treatment postretention outcomes compared using the American Board of Orthodontics objective grading system. Angle Orthod 2007;77:864-869.

12. Okunami TR, Kusnoto B, BeGole E, Evans CA, Sadowsky C, Fadavi S. Assessing the American Board of Orthodontics objective grading system: digital vs plaster dental casts. Am J Orthod Dentofacial Orthop 2007;131:51-56.

13. Detterline DA, Isikbay SC, Brizendine EJ, Kula KS. Clinical outcomes of 0.018-inch and 0.022-inch bracket slot using the ABO objective grading system. Angle Orthod 2010;80:528-532.

14. Jain M, Varghese J, Mascarenhas R, Mogra S, Shetty S, Dhakar N. Assessment of clinical outcomes of Roth and MBT bracket prescription using the American Board of Orthodontics Objective Grading System. Contemp Clin Dent 2013;4:307-312.

15. Knierim K, Roberts WE, Hartsfield J, Jr. Assessing treatment outcomes for a graduate orthodontics program: follow-up study for the classes of 2001-2003. Am J Orthod Dentofacial Orthop 2006;130:648-655, 655 e641-643.

16. Pinskaya YB, Hsieh TJ, Roberts WE, Hartsfield JK. Comprehensive clinical evaluation as an outcome assessment for a graduate orthodontics program. Am J Orthod Dentofacial Orthop 2004;126:533-543.

17. Paquette DE, Beattie JR, Johnston LE, Jr. A long-term comparison of nonextraction and premolar extraction edgewise therapy in "borderline" Class II patients. Am J Orthod Dentofacial Orthop 1992;102:1-14.

18. Konstantonis D. The impact of extraction vs nonextraction treatment on soft tissue changes in Class I borderline malocclusions. Angle Orthod 2012;82:209-217. 
19. Anthopoulou C, Konstantonis D, Makou M. Treatment outcomes after extraction and nonextraction treatment evaluated with the American Board of Orthodontics objective grading system. Am J Orthod Dentofacial Orthop 2014;146:717-723.

\section{Figures and tables}

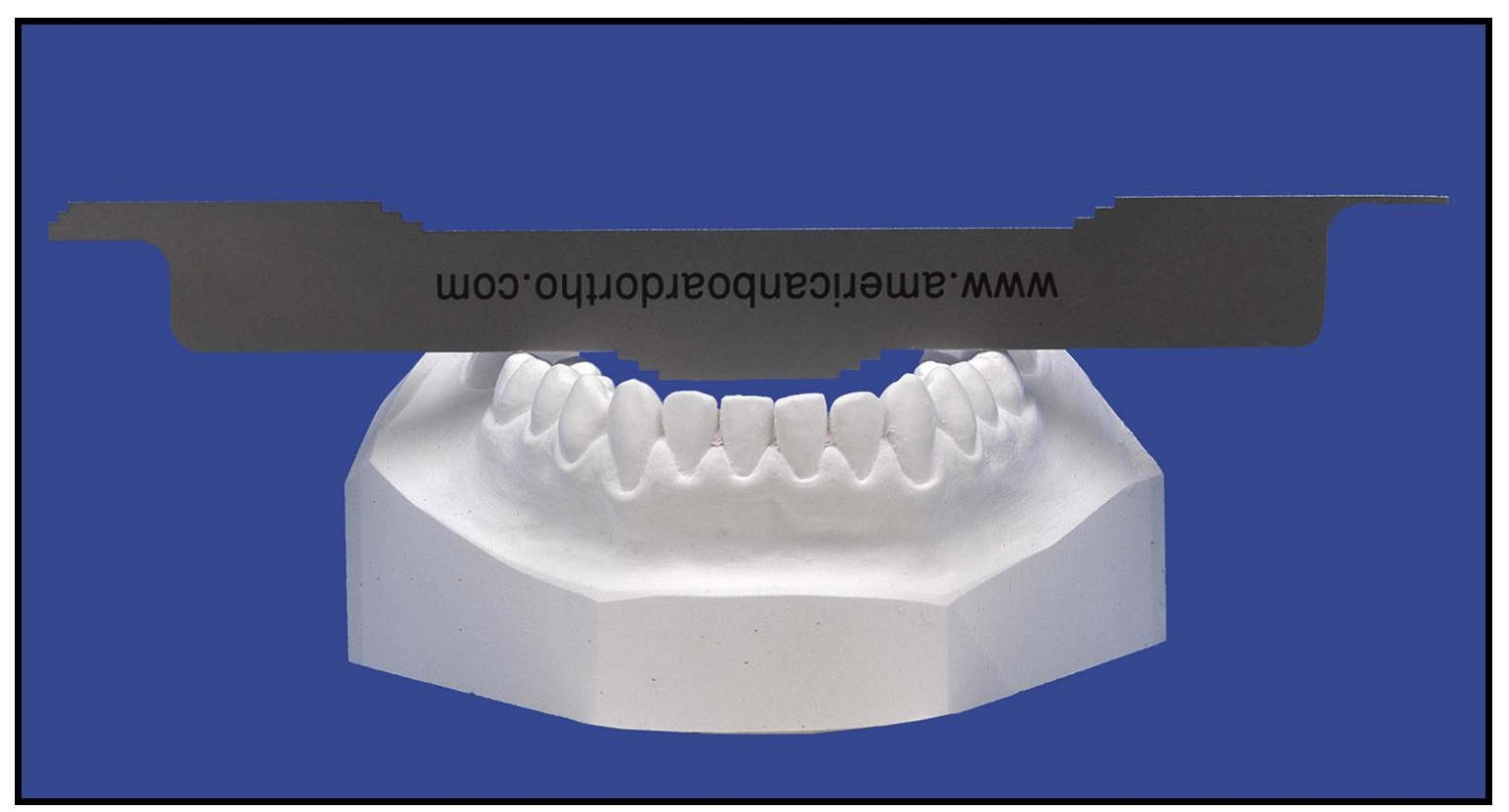

Fig 1. Use of the $A B O$ gauge on a plaster cast.

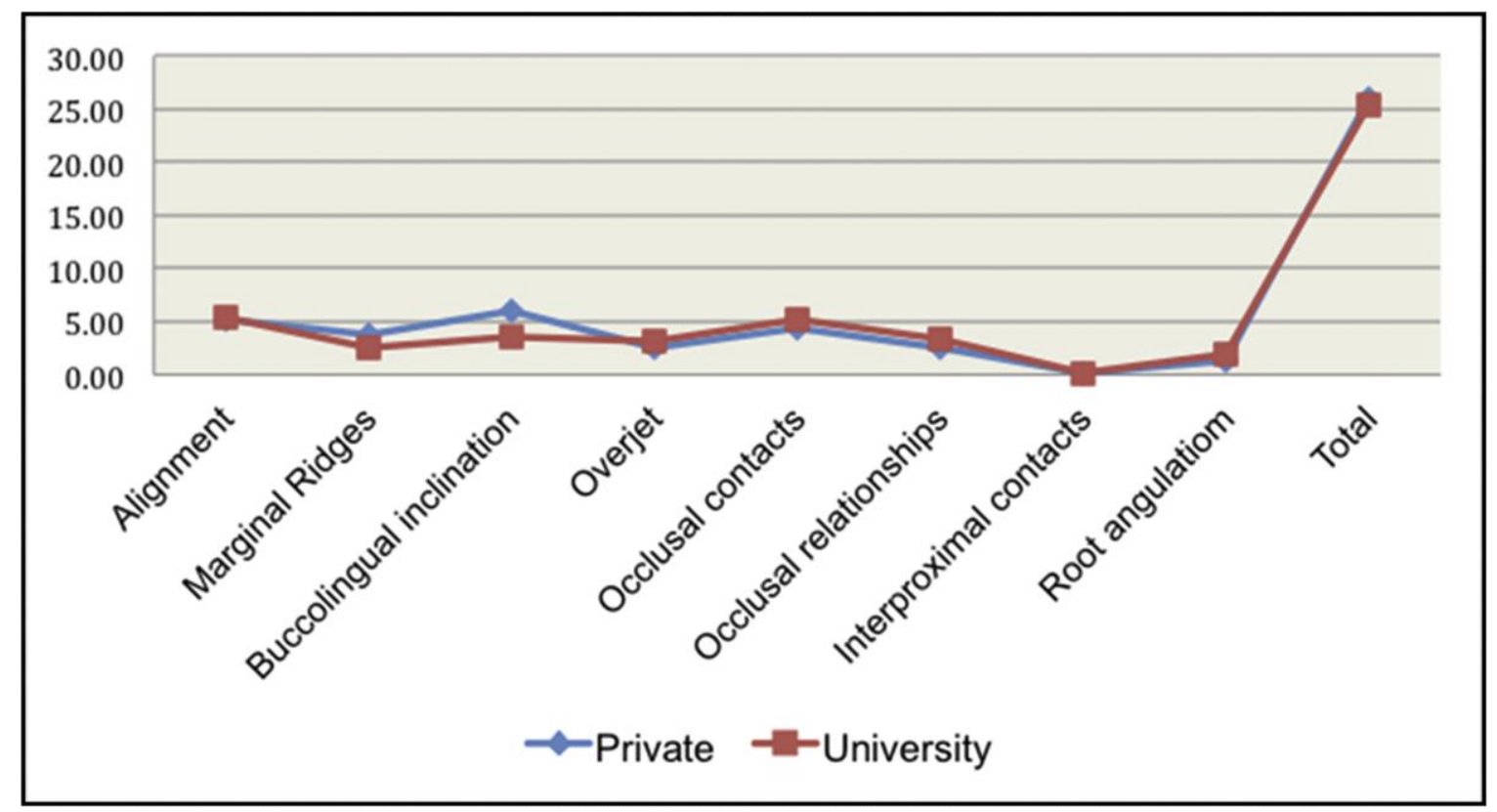

Fig 2. $A B O$ scores for the university and private practice groups for the $8 \mathrm{ABO}$ variables and for the total scores. 
Table 1. Descriptive statistics for age and gender of the sample.

\begin{tabular}{|c|c|c|c|c|c|c|c|c|}
\hline & & & \multicolumn{3}{|c|}{ Gender ${ }^{*}$} & \multicolumn{3}{|l|}{ Age } \\
\hline & & & Male & Female & Total & $\mathrm{N}$ & Mean & SD \\
\hline \multirow[t]{4}{*}{ Treatment } & Private & Count & 12 & 22 & 34 & \multirow[b]{2}{*}{34} & \multirow[b]{2}{*}{17.06} & \multirow[b]{2}{*}{7.87} \\
\hline & & \%within treatment & $35.3 \%$ & $64.7 \%$ & $100.0 \%$ & & & \\
\hline & \multirow[t]{2}{*}{ University } & Count & 13 & 19 & 32 & \multirow[b]{2}{*}{32} & \multirow[b]{2}{*}{14.88} & \multirow[b]{2}{*}{6.72} \\
\hline & & \%within treatment & $40.6 \%$ & $59.4 \%$ & $100.0 \%$ & & & \\
\hline \multirow[t]{2}{*}{ Total } & & Count & 25 & 41 & 66 & \multirow[b]{2}{*}{66} & \multirow[b]{2}{*}{16} & \multirow[b]{2}{*}{7.36} \\
\hline & & $\%$ within treatment & $37.9 \%$ & $62.1 \%$ & $100.0 \%$ & & & \\
\hline
\end{tabular}

${ }^{*}$ Fisher's exact test $p$-value $0.8{ }^{* *}$ t-test $p$-value 0.23

Table 2. Descriptive and inferential statistics for the eight ABO-OGS variables and for the total $\mathrm{ABO}-\mathrm{OGS}$ scores in the university group and in the private practice group.

\begin{tabular}{|c|c|c|c|c|c|c|c|c|}
\hline ABO-OGS Variable & Private & ractice (SD) & Univers & $y(S D)$ & Mean difference & $95 \% \mathrm{Cl}$ & & $p$-value ${ }^{*}$ \\
\hline Alignment & 5,12 & $(2,20)$ & 5,41 & $(3.47)$ & $-0,29$ & $-1,73$ & 1,16 & 0,69 \\
\hline Marginal Ridges & 3,85 & $(2,40)$ & 2,53 & $(1,76)$ & 1,32 & 0,28 & 2,36 & 0,01 \\
\hline Buccolingual inclination & 5,94 & $(3,43)$ & 3,66 & $(3,44)$ & 2,28 & 0,59 & 3,98 & 0,01 \\
\hline Overjet & 2,62 & $(2,17)$ & 3,16 & $(2,30)$ & $-0,54$ & $-1,64$ & 0,56 & 0,33 \\
\hline Occlusal contacts & 4,47 & $(3,65)$ & 5,25 & $(3,78)$ & $-0,78$ & $-2,61$ & 1,05 & 0,40 \\
\hline Occlusal relationships & 2,53 & $(2,78)$ & 3,38 & $(3,02)$ & $-0,85$ & $-2,27$ & 0,58 & 0,24 \\
\hline Interproximal contacts & 0,15 & $(0,44)$ & 0,16 & $(0,45)$ & $-0,01$ & $-0,23$ & 0,21 & 0,93 \\
\hline Root angulation & 1,26 & $(0,86)$ & 1,91 & $(1,51)$ & $-0,65$ & $-1,26$ & $-0,03$ & 0,04 \\
\hline Total & 25,94 & $(7,69)$ & 25,44 & $(9,81)$ & 0,5 & $-3,82$ & 4,82 & 0,82 \\
\hline
\end{tabular}

